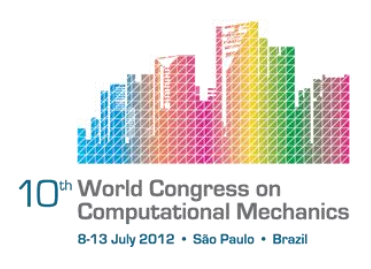

\title{
SIZE EFFECTS ON THE STRENGTH AND TOUGHNESS OF FIBRE-REINFORCED COMPOSITES
}

\author{
S. T. Pinho, S. Pimenta \\ Department of Aeronautics, Imperial College London (silvestre.pinho@imperial.ac.uk)
}

\begin{abstract}
This paper presents an analytical model for size effects on the longitudinal tensile strength of composite fibre bundles. The strength of individual fibres is modelled by a Weibull distribution, while the matrix (or fibre-matrix interface) is represented through a perfectlyplastic shear-lag model. A probabilistic analysis of the failure process in hierarchical bundles (bundles of bundles) is performed, so that a scaling law relating the strength distributions of consecutive bundle levels is derived. An efficient numerical scheme (based on asymptotic limits) is proposed, hence coupon-sized bundle strength distributions are obtained almost instantaneously. Parametric and sensitivity studies show that both fibre and matrix properties are critical for bundle strength; model predictions at different scales are validated against experimental results available in the literature.
\end{abstract}

Keywords: Size effect, Tensile strength, Hierarchical bundles, Fibre-reinforced composites.

\section{INTRODUCTION}

Size effects on the strength of composite materials are widely reported in the literature, but an universally accepted modelling strategy is still to be developed [1]; this represents a significant challenge for the design of large structures. This paper presents a model for size effects on the longitudinal tensile strength of UniDirectional (UD) Fibre-Reinforced Polymers (FRPs), based on the stochastic variability of fibre strength and the definition of hierarchical fibre-matrix bundles.

Several theories have been proposed to model the relation between size and strength of structures. The stochastic approach, based on the Weakest Link Theory (WLT) and formalised by Weibull [2], has been extensively applied to FRPs [1]. Deterministic size effects have also been studied by several other authors, e.g. Bazant et al. [3] (on the energy dissipated during failure of quasi-brittle materials), Carpinteri et al. [4] (on the fractal self-similar failure process of heterogeneous materials), and Wisnom et al. [5] (on the change of failure mode in scaled laminated composites). These authors acknowledge nevertheless that stochastic size effects play a major role in the strength of FRPs.

The WLT states that a chain withstands an external load only if all its elements survive the resulting stresses. Let $X_{\mathrm{U}, \mathrm{r}}$ be the stochastic strength of the elements (with reference length $l_{\mathrm{r}}$, all statistically identical and independent) under an uniform (subscript U) stress $\sigma$; 


\begin{tabular}{|lll|}
\hline \multicolumn{1}{|c|}{ Nomenclature } & \multicolumn{2}{l}{ Superscripts } \\
$A$ Uppercase variables & cross sectional area & f fibre \\
$C$ & perimeter & {$[i]$ bundle level } \\
$\mathcal{C}_{\mathrm{K}}$ & stress-concentrations strength parameter & $\infty$ remote \\
$\mathrm{CoV}$ & coefficient of variation of strength & \\
$\Gamma$ & shear-lag boundary & \multicolumn{2}{l}{ Subscripts } \\
$F$ & failure probability (strength distribution) & 0 Weibull scale parameter \\
$S$ & survival probability & $\mathrm{B}$ free edges boundary \\
$T$ & shear strength & $\mathrm{C} \quad$ control length \\
$V$ & volume fraction & $\mathrm{e}$ effective recovery length \\
$X$ & longitudinal tensile strength & $\mathrm{H}$ hexagonal configuration \\
Lowercase variables & $\mathrm{I}$ interface failure path \\
$f$ & strength probability density function & $\mathrm{K}$ linear stress concentrations state \\
$\phi$ & diameter & $\mathrm{L}$ pure linear stress state \\
$i$ & bundle level & $\mathrm{M}$ matrix failure path \\
$k$ & stress concentrations factor & $\mathrm{m}$ mean value \\
$l$ & length & $\mathrm{n}$ chain of elements \\
$m$ & Weibull shape parameter & $\mathrm{Q}$ quadrangular configuration \\
$n$ & number of elements & $\mathrm{r}$ reference length / element \\
$s$ & interfibre spacing & $\mathrm{S}$ smallest failure path \\
$\sigma$ & longitudinal stress & $\mathrm{SL}$ shear-lag \\
$\tau$ & shear stress & $\mathrm{U}$ uniform stress state \\
\hline
\end{tabular}

the survival probability for each element is $S_{\mathrm{U}, \mathrm{r}}(\sigma)=\operatorname{Pr}\left(X_{\mathrm{U}, \mathrm{r}}>\sigma\right)$. Therefore, the survival probability of a chain (length $l_{\mathrm{n}}=n \cdot l_{\mathrm{r}}$ ) with $n$ elements is:

$$
S_{\mathrm{U}, \mathrm{n}}(\sigma)=\left[S_{\mathrm{U}, \mathrm{r}}(\sigma)\right]^{n} \quad \Rightarrow \quad \ln \left[S_{\mathrm{U}, \mathrm{n}}(\sigma)\right]=n \cdot \ln \left[S_{\mathrm{U}, \mathrm{r}}(\sigma)\right]=\frac{l_{\mathrm{n}}}{l_{\mathrm{r}}} \cdot \ln \left[S_{\mathrm{U}, \mathrm{r}}(\sigma)\right] .
$$

Weibull [2] then proposed a new distribution for the strength of brittle materials, so that survival and failure probabilities of a chain under uniform stresses $\sigma$ are:

$$
S_{\mathrm{U}, \mathrm{n}}(\sigma)=\exp \left[-\frac{l_{\mathrm{n}}}{l_{\mathrm{r}}}\left(\frac{\sigma}{\sigma_{0}}\right)^{m}\right] \quad \text { and } \quad F_{\mathrm{U}, \mathrm{n}}(\sigma)=1-\exp \left[-\frac{l_{\mathrm{n}}}{l_{\mathrm{r}}}\left(\frac{\sigma}{\sigma_{0}}\right)^{m}\right],
$$

where $m$ and $\sigma_{0}$ are respectively the shape (size independent) and scale (measured at $l_{r}$ ) parameters of the distribution. A true Weibull distribution $F_{\mathrm{U}, \mathrm{n}}(\sigma)$ is linear in Weibull coordinates, defined as $\ln (\sigma)$ vs. $\ln \left[-\ln \left(1-F_{\mathrm{U}, \mathrm{n}}\right)\right]$.

Equation 2 has been widely used to model the length effect on the strength of technical fibres, e.g. glass or carbon [5-7]. While alternative strength distributions have been proposed [8,9], a good agreement between Weibull's theory and experimental measurements can be achieved if the spurious effect of fibre gripping and testing variability is taken into account [10].

At the FRP level, the parallel fibre arrangement and the presence of matrix result in a quasi-brittle failure process; this hinders the applicability of Weibull's theory to model size effects on the longitudinal tensile strength of FRPs [1,3], especially when scaling the cross-section or filament count. Beyerlein and Phoenix [9] and Kazanci [11] tested different micro-bundles (with 4 and 7 fibres) and found that bundle strengths deviated significantly from Weibull distributions; moreover, the mean strength of some bundles (depending on the resin) was higher than that of the fibres, while variability was considerably lower. At the 
macroscopic scale, Okabe and Takeda [12] and Scott et al. [13] reported the formation of several clusters of fibre breaks before final coupon failure. It has also been suggested that both the magnitude of size effects and the variability of strength decrease for larger specimens [1]. All these observations at the FRP level are incompatible with the WLT.

The asymptotic behaviour for the tensile strength of FRPs has been successfully modelled in the literature $[1,3]$. However, the recent development of high-performance composites with discontinuous reinforcement - with individual fibres and bundles of various sizes $[14,15]$ - requires developing and validating full scaling models. It has been suggested [1] that most of the physics involved in longitudinal tensile failure of FRPs are captured by fibre bundle models, first developed by Daniels [16]; however, and despite many developments [7], no single approach has surfaced as providing simultaneously a definitive explanation for the micromechanics and statistics of fibre-dominated tensile failure, as well as validated quantitative predictions for size effects over a complete range of scales.

Pimenta et al. [15] and Laffan et al. [17] reported self-similar or quasi-fractal features on the fracture surface of thin (under $0.5 \mathrm{~mm}$ ) UD laminas and fibre bundles, which suggests a hierarchical failure process. Such features had already been proposed in Newman and Gabrielov's model [18] for dry fibre bundles. Considering that a bundle of hierarchical level $[i+1]$ is composed by two sub-bundles of level $[i]$, the following recursive relation for bundle strength distributions was derived [18]:

$$
F^{[i+1]}(\sigma)=F^{[i]}(\sigma) \cdot\left[2 \cdot F^{[i]}(2 \cdot \sigma)-F^{[i]}(\sigma)\right],
$$

where $F^{[i]}(\sigma)$ is the failure probability of a level- $[i]$ bundle under an applied stress $\sigma$. Newman and Gabrielov's model provides a physically sound and computationally robust alternative to the WLT. However, it does not consider the effect of an embedding matrix, and does not include any characteristic length governing the failure process (which is paramount for quasibrittle materials [3]); moreover, the model is inconsistent with the WLT for length scaling, and no alternative is proposed.

This paper presents the development, implementation and validation of a fibre-bundle model for predicting size effects on the longitudinal tensile strength of FRP (embedded) bundles. Following Newman and Gabrielov's work [18], bundles are hierarchically organised and scaling is based on the single-fibre strength distribution. However, the role of the matrix (or fibre-matrix interface) is now considered through a simplified shear-lag model, with substantial implications on the derivation of the scaling law and its implementation.

This paper is organised as follows: Section 2 presents the development of the analytical model for predicting strength distributions of FRP bundles of different dimensions. Section 3 explores modelling results (including sensitivity studies and experimental validation), which are subsequently interpreted and discussed in Section 4. Finally, Section 5 draws the main conclusions.

\section{MODEL DEVELOPMENT}

\subsection{Methodology and definitions}

This model is based on hierarchical fibre-matrix bundles (Figure 1(a)). Their geometry is defined in Section 2.2, with particular emphasis on the shear-lag boundary $\left(\Gamma_{\mathrm{SL}}\right)$, over 


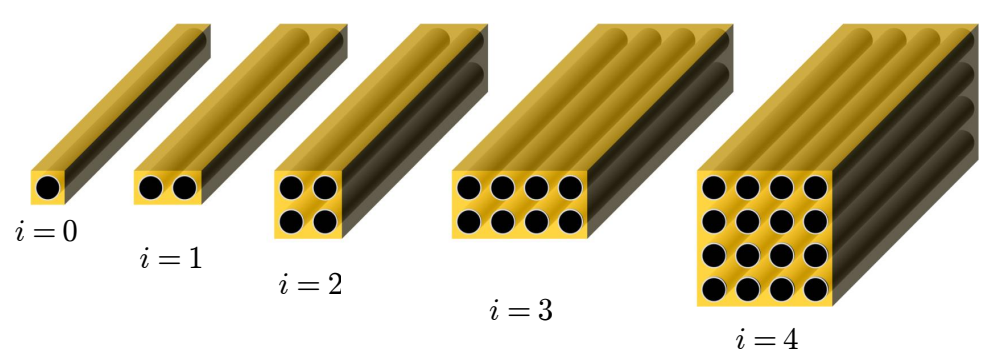

(a) Bundle hierarchy.

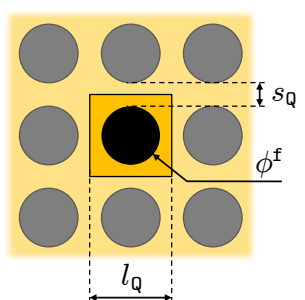

(b) Fibre arrangement.

Figure 1. Hierarchical bundles in quadrangular fibre arrangement.

which a shear-lag model will include the effect of the matrix or fibre-matrix interface on the failure process. The in-situ mechanical response of the matrix in a composite is difficult to represent accurately; for instance, epoxy is usually brittle in bulk, but actually ductile and much stronger in-situ $[19,20]$. A perfectly-plastic constitutive law, with shear strength $T_{\mathrm{SL}}$, is adopted for the sake of simplicity.

Considering that this shear-lag model yields non-uniform stress fields near fibre breaks (with linear stress concentrations), the corresponding failure probabilities are derived in Section 2.3. The amplitude of true stress concentrations near clusters of fibre breaks is still an unsolved issue [20-22]; in this model, the stress concentrations factor $k$ is a parameter assumed to be constant throughout bundle hierarchy.

Section 2.4 derives the hierarchical law for bundle failure. This requires (i) identifying the stress fields and sequences of events leading to failure of a 2-fibres bundles, and (ii) calculating the respective bundle strength distribution. A self-similar failure process is then assumed $[15,17]$, so the previous result is used recursively throughout bundle hierarchy. The numerical implementation of the model is described in Section 2.5.

This paper expresses longitudinal stresses $(\sigma)$ as fibre stresses (i.e. normalised by the area of fibres in the cross section). The concept of stochastic strength under uniform stresses will be extended to proportional non-uniform stress fields, characterised by a shape function $\Phi$ and remote stress $\sigma^{\infty}$. Under such stress field, the strength of an element $\mathcal{E}$ with length $\ell$ is represented as $X_{\Phi, \ell}^{\mathcal{E}}$, and failure occurs if $X_{\Phi, \ell}^{\mathcal{E}} \leq \sigma^{\infty}$.

The failure probability (cumulative distribution function) of the element $\mathcal{E}$ of length $\ell$ under the stress field $\Phi \cdot \sigma^{\infty}$ is $F_{\Phi, \ell}^{\mathcal{E}}\left(\sigma^{\infty}\right)=\operatorname{Pr}\left(X_{\Phi, \ell}^{\mathcal{E}} \leq \sigma^{\infty}\right)$; this corresponds to the traditional strength distribution for uniform stresses. The associated survival probability is $S_{\Phi, \ell}^{\mathcal{E}}\left(\sigma^{\infty}\right)=$ $1-F_{\Phi, \ell}^{\mathcal{E}}\left(\sigma^{\infty}\right)$, and the probability density function is $f_{\Phi, \ell}^{\mathcal{E}}\left(\sigma^{\infty}\right)=\mathrm{d} F_{\Phi, \ell}^{\mathcal{E}}\left(\sigma^{\infty}\right) / \mathrm{d} \sigma^{\infty}$.

\subsection{Fibre bundle geometry and shear-lag boundary}

Figure 1(a) illustrates the generation of hierarchical fibre bundles; this consists on pairing individual fibres (level-[0]) into level-[1] bundles, and then sequentially grouping two level- $[i]$ bundles into one level- $[i+1]$ bundle [18]. The number of fibres $\left(n^{f}\right)$ in a level- $[i]$ bundle is therefore:

$$
n^{\mathrm{f}}=2^{i} \quad \Leftrightarrow \quad i=\log _{2} n^{\mathrm{f}} .
$$

The fibres (superscript $f$ ) in bundles are assumed to be arranged in a quadrangular architecture (subscript Q). The composite is characterised by the fibre diameter $\phi^{\text {f }}$ (circum- 

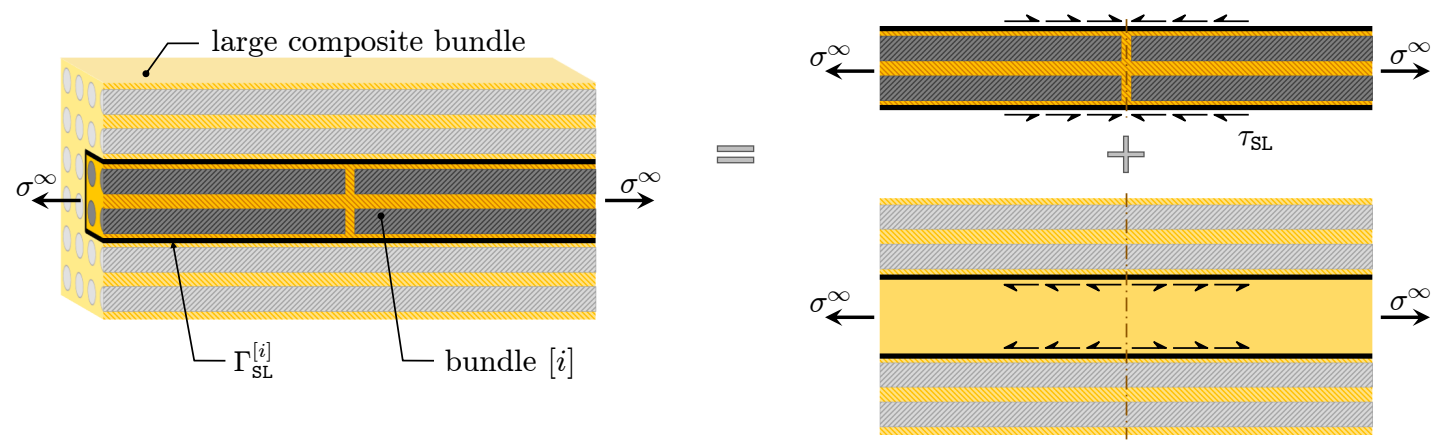

Figure 2. Shear-lag boundary.

ference $C^{\mathrm{f}}$ and area $A^{\mathrm{f}}$ ) and fibre volume fraction $V^{\mathrm{f}}$; the side length of the unit cell $\left(l_{\mathrm{Q}}\right)$ and interfibre spacing $\left(s_{\mathrm{Q}}\right)$ are (Figure 1(b)):

$$
l_{\mathrm{Q}}=\frac{\sqrt{\pi} \cdot \phi^{\mathrm{f}}}{2 \cdot \sqrt{V^{\mathrm{f}}}} \quad \text { and } \quad s_{\mathrm{Q}}=\left(\frac{\sqrt{\pi}}{2 \cdot \sqrt{V^{\mathrm{f}}}}-1\right) \cdot \phi^{\mathrm{f}} .
$$

Considering now a level- $[i]$ bundle embedded in a larger composite bundle (Figure 2), it is possible to define a level- $[i]$ shear-lag boundary $\left(\Gamma_{\mathrm{SL}}^{[i]}\right)$. Physically, $\Gamma_{\mathrm{SL}}^{[i]}$ corresponds to the surface at which shear-lag stresses will be transferred between the (unbroken) surrounding material and a broken level- $[i]$ bundle.

Table 1 presents the perimeter of the shear-lag boundary $\left(C^{[i]}\right)$ as function of $n^{\mathrm{f}}$, for three simplified geometries (corresponding to different relations between the strengths of matrix and fibre-matrix interface). The expressions shown are strictly valid only for even values of $i$, but used for any bundle size so that $C^{[i]}$ is a smooth function of $n^{\mathrm{f}}$.

Alternative configurations for the shear-lag boundary can also be defined [23]. Table 2 takes into account the effect of free boundaries in the large embedding bundle (level $[i=$ $\left.i^{\max }\right]$ ), by defining edge and corner boundaries with reduced effective perimeters. In addition, considering a hexagonal fibre packing generates fractal shear-lag boundaries (Table 3).

\subsection{Survival probabilities under several loading conditions}

\subsubsection{Generalisation of the weakest link theory for non-uniform stress fields}

The WLT (Equation 1) can be generalised to non-uniform chain stresses. Consider that each reference element $j=\{1 \ldots n\}$, of length $l_{\mathrm{r}}$, is under a uniform tensile stress $\sigma_{j}$. The chain (of length $l_{\mathrm{n}}=n \cdot l_{\mathrm{r}}$ ) is subjected to a piecewise constant but otherwise generic stress field with shape function $\Phi$, with a log-survival probability related to that of the uniformly loaded chain (subscript U):

$$
S_{\Phi, \mathrm{n}}=\prod_{j=1}^{n} S_{\mathrm{U}, \mathrm{r}}\left(\sigma_{j}\right) \stackrel{\text { Eq. } 1}{\Longrightarrow} \ln \left[S_{\Phi, \mathrm{n}}\right]=\sum_{j=1}^{n} \frac{l_{\mathrm{r}}}{l_{\mathrm{n}}} \cdot \ln \left[S_{\mathrm{U}, \mathrm{n}}\left(\sigma_{j}\right)\right] .
$$

This relation will be applied to chains under linear stress fields (Figure 3). 
Table 1. Shear-lag perimeter in quadrangular arrangement, derived for even values of $i$.

$\begin{aligned} & \text { Matrix } \\ & \text { failure } \\ & \text { (subscript } \\ & \text { QM) }\end{aligned}$
$\begin{gathered}\text { Interface } \\ \text { failure } \\ \text { (subscript } \\ \text { QI) }\end{gathered}$
$\begin{gathered}\text { Smallest } \\ \text { boundary } \\ \text { (subscript } \\ \text { QS) }\end{gathered}$

Table 2. Shear-lag perimeter considering boundary effects, derived for even values of $i^{\max }$.

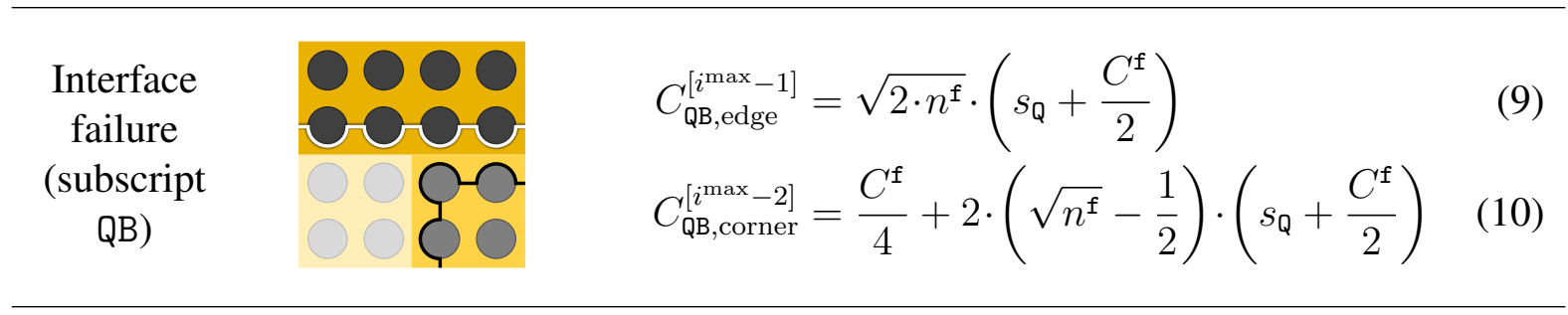

Table 3. Shear-lag perimeter in hexagonal arrangement, derived for $n^{\mathrm{f}}=\{1,7,49, \ldots\}$.

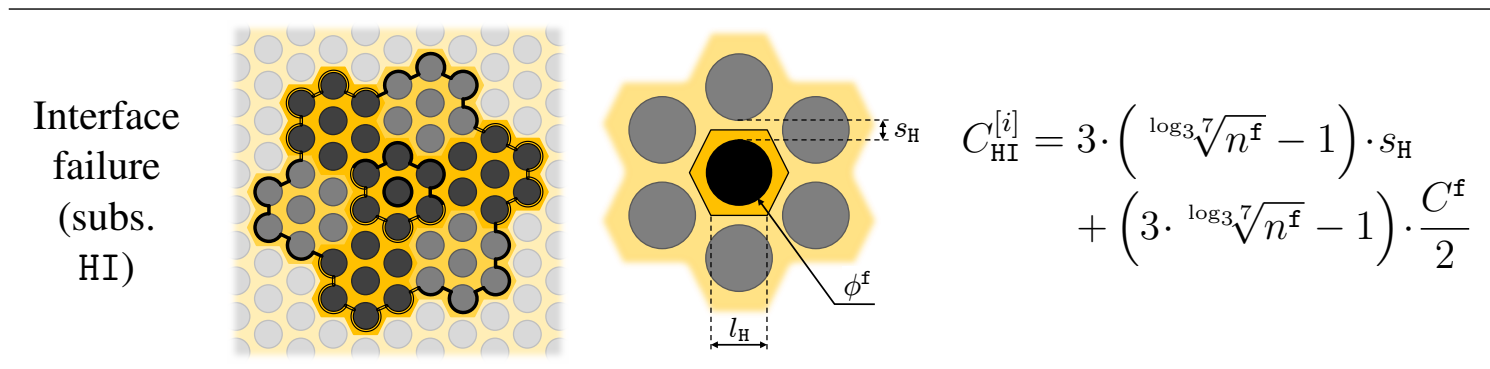

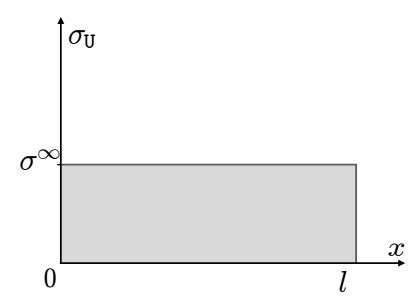

(a) Uniform loading.

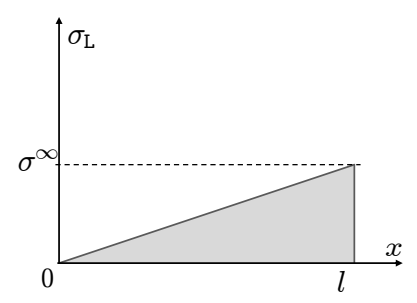

(b) Pure linear loading.

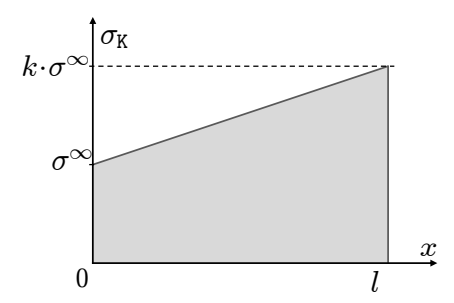

(c) Linear stress concentrations.

Figure 3. Stress fields analysed through a generalised weakest link theory. 


\subsubsection{Pure linear loading}

Consider a chain of length $l$ under a pure linear tensile stress field (subscript L, Figure 3(b)):

$$
\sigma_{\mathrm{L}}(x)=\frac{\sigma^{\infty}}{l} \cdot x \quad, \quad x \in[0, l] .
$$

Dividing the linearly-loaded chain into $n \rightarrow \infty$ links of individual length $\Delta x=l / n$, and following Equation 12, the survival probability $\left(S_{\mathrm{L}}\right)$ of the chain under $\sigma_{\mathrm{L}}(x)$ relates to that of an uniformly loaded chain $\left(S_{\mathrm{U}}\right)$ by:

$$
\ln \left[S_{\mathrm{L}}\right]=\lim _{n \rightarrow \infty} \sum_{j=1}^{n} \frac{\Delta x}{l} \cdot \ln \left[S_{\mathrm{U}}\left(\sigma_{\mathrm{L}}\left(x_{j}\right)\right)\right] .
$$

From Riemann's integral definition, and changing the integration variable from $x$ to $\sigma_{\mathrm{L}}$,

$$
\ln \left[S_{\mathrm{L}}\left(\sigma^{\infty}\right)\right]=\frac{1}{\sigma^{\infty}} \int_{\sigma_{\mathrm{L}}=0}^{\sigma^{\infty}} \ln \left[S_{\mathrm{U}}\left(\sigma_{\mathrm{L}}\right)\right] \mathrm{d} \sigma_{\mathrm{L}}
$$

Equation 15 is valid regardless of the shape of the material strength distribution. For the particular case of a Weibull distribution (Equation 2),

$$
\begin{aligned}
& \ln \left[S_{\mathrm{L}}\left(\sigma^{\infty}\right)\right]=\frac{1}{\sigma^{\infty}} \int_{\sigma_{\mathrm{L}}=0}^{\sigma^{\infty}}-\left(\frac{\sigma_{\mathrm{L}}}{\sigma_{0}}\right)^{m} \mathrm{~d} \sigma_{\mathrm{L}}=-\frac{1}{m+1}\left(\frac{\sigma^{\infty}}{\sigma_{0}}\right)^{m}=\frac{1}{m+1} \cdot \ln \left[S_{\mathrm{U}}\left(\sigma^{\infty}\right)\right] \\
& \Rightarrow \quad F_{\mathrm{L}}\left(\sigma^{\infty}\right)=1-\exp \left[-\frac{1}{1+m}\left(\frac{\sigma^{\infty}}{\sigma_{0}}\right)^{m}\right] \text {. }
\end{aligned}
$$

\subsubsection{Linear stress concentrations}

Consider a chain of length $l$ under a linear stress concentrations field with factor $k$ (subscript K, Figure 3(c)):

$$
\sigma_{\mathrm{K}}(x)=\sigma^{\infty}+\frac{\sigma^{\infty} \cdot(k-1)}{l} \cdot x \quad, \quad x \in[0, l] .
$$

Using the same procedure followed for the pure linear stresses case, the survival probability $\left(S_{\mathrm{K}}\right)$ of the chain loaded with linear stress concentrations is defined as:

$$
\ln \left[S_{\mathrm{K}}\left(\sigma^{\infty}\right)\right]= \begin{cases}\frac{1}{\sigma^{\infty} \cdot(k-1)} \int_{\sigma_{\mathrm{K}}=\sigma^{\infty}}^{k \cdot \sigma^{\infty}} \ln \left[S_{\mathrm{U}}\left(\sigma_{\mathrm{K}}\right)\right] \mathrm{d} \sigma_{\mathrm{K}} & \text { if } \quad k>1 \\ \ln \left[S_{\mathrm{U}}\left(\sigma^{\infty}\right)\right] & \text { if } \quad k=1 .\end{cases}
$$

Equations 15 and 18 can be combined to establish a relation between survival probabilities of chains under pure linear stresses and under linear stress concentrations:

$$
\ln \left[S_{\mathrm{K}}\left(\sigma^{\infty}\right)\right]=\left\{\begin{array}{lll}
\frac{k \cdot \ln \left[S_{\mathrm{L}}\left(k \cdot \sigma^{\infty}\right)\right]-\ln \left[S_{\mathrm{L}}\left(\sigma^{\infty}\right)\right]}{k-1} & \text { if } \quad k>1 \\
\ln \left[S_{\mathrm{U}}\left(\sigma^{\infty}\right)\right] & \text { if } \quad k=1
\end{array}\right.
$$


Equations 18 and 19 are valid regardless of the shape of the material strength distribution. For the particular case of a Weibull distribution (Equation 2):

$$
\begin{gathered}
\ln \left[S_{\mathrm{K}}\left(\sigma^{\infty}\right)\right]=\frac{1}{\sigma^{\infty} \cdot(k-1)} \int_{\sigma_{\mathrm{K}}=\sigma^{\infty}}^{k \cdot \sigma^{\infty}}-\left(\frac{\sigma_{\mathrm{K}}}{\sigma_{0}}\right)^{m} \mathrm{~d} \sigma_{\mathrm{K}}=-\mathcal{C}_{\mathrm{K}}\left(\frac{\sigma^{\infty}}{\sigma_{0}}\right)^{m}=\mathcal{C}_{\mathrm{K}} \cdot \ln \left[S_{\mathrm{U}}\left(\sigma^{\infty}\right)\right] \\
\Rightarrow \quad F_{\mathrm{K}}\left(\sigma^{\infty}\right)=1-\exp \left[-\mathcal{C}_{\mathrm{K}} \cdot\left(\frac{\sigma^{\infty}}{\sigma_{0}}\right)^{m}\right], \quad \text { where } \quad \mathcal{C}_{\mathrm{K}}=\frac{k^{m+1}-1}{(m+1)(k-1)}
\end{gathered}
$$

\subsection{Hierarchical law for bundle failure}

\subsubsection{Stress field around a fibre break and definition of the control region}

Consider a level-[1] bundle of reference length $l_{r}$ (subscript $r$ ), composed by two level-[0] fibres $-\mathcal{A}$ and $\mathcal{B}-$ embedded in a soft matrix (Figure $1(\mathrm{a}), i=1$ ). The bundle is loaded in tension by a progressively increasing remote stress $\sigma^{\infty}$, so that each fibre undergoes a uniform stress state $\sigma^{\mathcal{A}}(x)=\sigma^{\mathcal{B}}(x)=\sigma^{\infty}$.

Assume that, for a given $\sigma^{\infty}$, fibre $\mathcal{A}$ is failed at the location $x=0$ (Figure 4(a)). Following a perfectly-plastic shear-lag model (with strength $T_{\mathrm{SL}}$ and shear-lag perimeter $C^{[0]}$, defined in Tables 1-2), fibre $\mathcal{A}$ will recover linearly the longitudinal remote stresses within the level-[0] effective recovery length (subscript e), defined as:

$$
l_{\mathrm{e}}^{[0]}\left(\sigma^{\infty}\right)=2 \cdot \frac{A^{\mathrm{f}}}{C^{[0]} \cdot T_{\mathrm{SL}}} \cdot \sigma^{\infty} .
$$

Conversely, fibre $\mathcal{B}$ will undergo linear stress concentrations ( $k=2$ from equilibrium) within the length $l_{\mathrm{e}}^{[0]}$ (Figure 4(a)).

Bundle failure requires that both fibres $\mathcal{A}$ and $\mathcal{B}$ break in nearby locations, so as to promote complete yielding of the shear-lag boundary (representing the matrix or interface) as shown in Figure 4(b). Therefore, once fibre $\mathcal{A}$ fails, the level-[1] control region - within which a break in fibre $\mathcal{B}$ leads to bundle failure - is confined to the control length (subscript c), defined by:

$$
l_{\mathrm{c}}^{[1]}\left(\sigma^{\infty}\right)=2 \cdot l_{\mathrm{e}}^{[0]}\left(\sigma^{\infty}\right)=4 \cdot \frac{A^{\mathrm{f}}}{C^{[0]} \cdot T_{\mathrm{SL}}} \cdot \sigma^{\infty} .
$$

The control region (centred at the first fibre break) is partitioned into 4 fibre segments $\left(\mathcal{A}_{1}, \mathcal{A}_{2}, \mathcal{B}_{1}\right.$ and $\left.\mathcal{B}_{2}\right)$ of equal length $l_{\mathrm{e}}^{[0]}$ (Figure $4(\mathrm{c})$ ). Fibre strength under uniform stresses is assumed to follow a Weibull distribution, with parameters $m$ and $\sigma_{0}^{\mathrm{f}}$ at $l_{\mathrm{r}}$, and survival probability $S_{\mathrm{U}, \mathrm{r}}^{[0]}$. Scaling for the segments $\mathcal{A}_{1}, \mathcal{A}_{2}, \mathcal{B}_{1}$ and $\mathcal{B}_{2}$ of length $l_{\mathrm{e}}^{[0]}$, under uniform $\left(S_{\mathrm{U}, \mathrm{e}}^{[0]}\right)$ or stress concentrations $\left(S_{\mathrm{K}, \mathrm{e}}^{[0]}\right)$ fields, can be done through Equations 2 and 20, leading to:

$$
S_{\mathrm{U}, \mathrm{r}}^{[0]}=\exp \left[-\left(\frac{\sigma^{\infty}}{\sigma_{0}^{\mathrm{f}}}\right)^{m}\right], \quad S_{\mathrm{U}, \mathrm{e}}^{[0]}=\exp \left[-\frac{l_{\mathrm{e}}^{[0]}}{l_{\mathrm{r}}}\left(\frac{\sigma^{\infty}}{\sigma_{0}^{\mathrm{f}}}\right)^{m}\right], \quad S_{\mathrm{K}, \mathrm{e}}^{[0]}=\exp \left[-\mathcal{C}_{\mathrm{K}} \frac{l_{\mathrm{e}}^{[0]}}{l_{\mathrm{r}}}\left(\frac{\sigma^{\infty}}{\sigma_{0}^{\mathrm{f}}}\right)^{m}\right] .
$$

The stochastic strength of each element is represented following a similar notation (e.g. $X_{\mathrm{K}, \mathrm{e}}^{\mathcal{B}_{1}}$ is the strength of element $\mathcal{B}_{1}$ of length $l_{\mathrm{e}}$ under linear stress concentrations). 


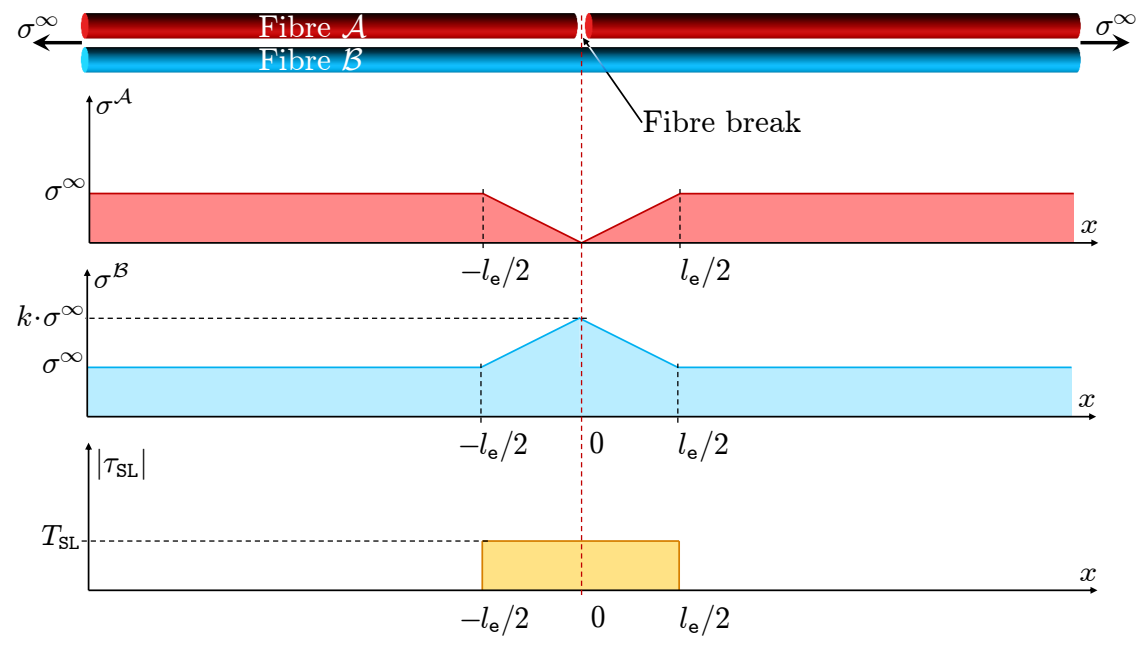

(a) Stress fields after first fibre failure.

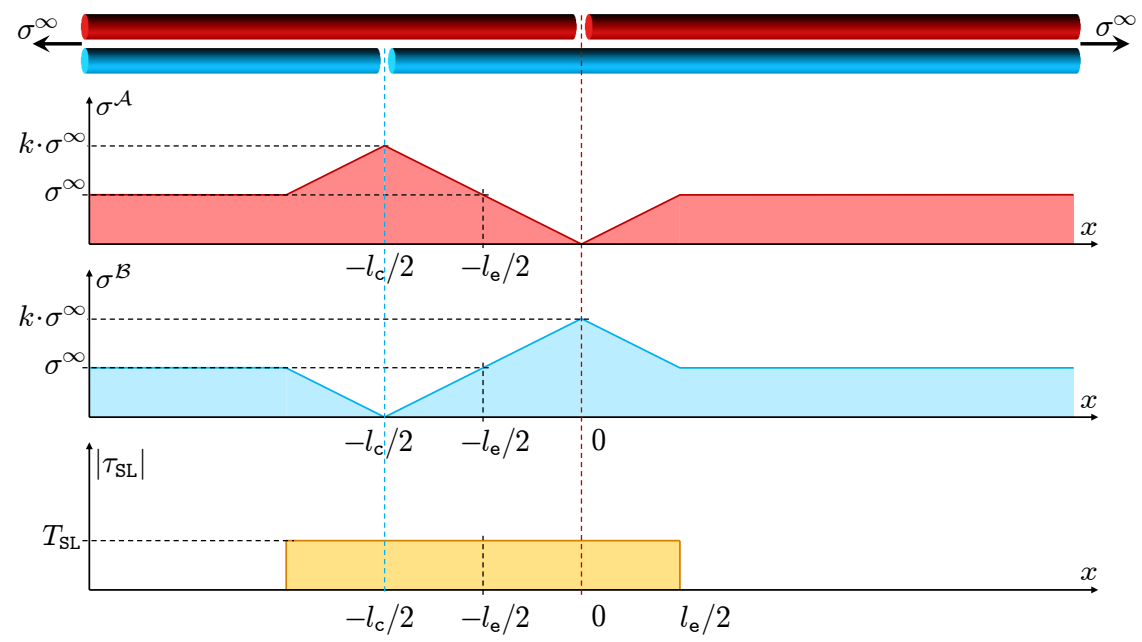

(b) Definition of critical distance between fibre breaks: the bundle fails only if fibre $\mathcal{B}$ breaks at a distance smaller than $l_{\mathrm{c}} / 2$ from the break in fibre $\mathcal{A}$.

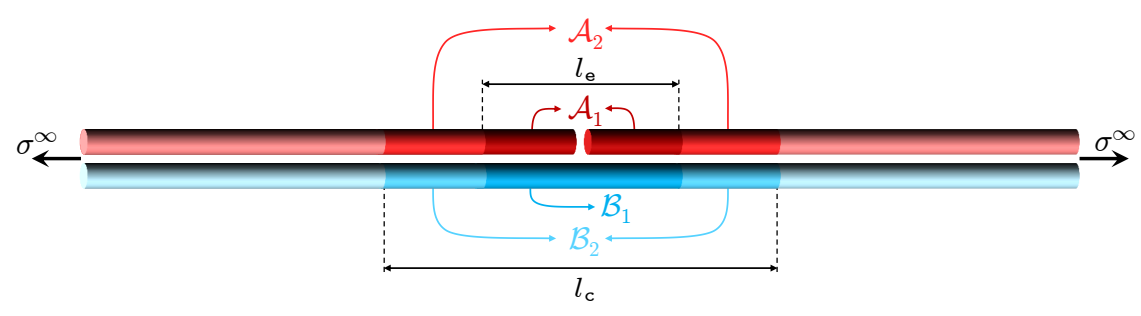

(c) Definition of the control region and fibre segments.

Figure 4. Stress fields and length scales in a level-[1] fibre bundle. 


\subsubsection{Recursive law for bundle failure}

Considering the bundle analysed in Figure 4 and assuming that:

(i) at each remote stress $\sigma^{\infty}$, the bundle is represented by a chain of independent control regions of length $l_{\mathrm{c}}^{[1]}$ (Equation 22).

(ii) within each control region, each fibre can break only once (equivalent to the WLT), the bundle survival probability is defined as:

$$
S_{\mathrm{U}, \mathrm{c}}^{[1]}\left(\sigma^{\infty}\right)=S_{\mathrm{U}, \mathbf{e}}^{[0]}\left(\sigma^{\infty}\right)^{4}+2 \cdot\left[1-S_{\mathrm{U}, \mathbf{e}}^{[0]}\left(\sigma^{\infty}\right)^{2}\right] \cdot S_{\mathrm{U}, \mathbf{e}}^{[0]}\left(\sigma^{\infty}\right) \cdot S_{\mathrm{K}, \mathbf{e}}^{[0]}\left(\sigma^{\infty}\right)
$$

Physically, Equation 24 states that the bundle survives either if all its 4 segments survive, or if the weakest fibre fails and the strongest one survives the resulting stress field. It can be demonstrated [23] that Equation 24 considers three possible failure modes: (i) unstable bundle failure, (ii) stable bundle failure due to stress concentrations, and (iii) stable bundle failure due to independent fibre flaws.

Assuming a self-similar hierarchical failure process, Equation 24 can be extrapolated to any bundle level. The survival function of a level- $[i+1]$ bundle (under uniform stresses and within a control length, omitting $\sigma^{\infty}$ for readability) is thus calculated as:

$$
S_{\mathrm{U}, \mathrm{c}}^{[i+1]}=\left(S_{\mathrm{U}, \mathrm{e}}^{[i]}\right)^{4}+2 \cdot\left[1-\left(S_{\mathrm{U}, \mathrm{e}}^{[i]}\right)^{2}\right] \cdot S_{\mathrm{U}, \mathrm{e}}^{[i]} \cdot S_{\mathrm{K}, \mathrm{e}}^{[i]}
$$

Level- $[i]$ survival probabilities are defined at the effective recovery length $l_{\mathrm{e}}^{[i]}\left(\sigma^{\infty}\right)$ (the shearlag perimeter $C^{[i]}$ can be calculated from Equations 6-10), while $S_{\mathrm{U}, \mathrm{c}}^{[i+1]}$ is defined at the level$[i+1]$ control length:

$$
l_{\mathrm{e}}^{[i]}\left(\sigma^{\infty}\right) \stackrel{\text { Eq. } 21}{=} 2 \cdot \frac{n^{\mathrm{f}} \cdot A^{\mathrm{f}}}{C^{[i]} \cdot T_{\mathrm{SL}}} \cdot \sigma^{\infty} \quad, \quad l_{\mathrm{c}}^{[i+1]}\left(\sigma^{\infty}\right) \stackrel{\text { Eq. } 22}{=} 2 \cdot l_{\mathrm{e}}^{[i]}\left(\sigma^{\infty}\right) .
$$

Equation 25 can be scaled to the reference length (following Equation 1 and 26) and written in both the following logarithmic forms:

$$
\begin{aligned}
\ln \left(S_{\mathrm{U}, \mathrm{r}}^{[i+1]}\right) & =2 \cdot \ln \left(S_{\mathrm{U}, \mathrm{r}}^{[i]}\right)+\frac{l_{\mathrm{r}}}{2 \cdot l_{\mathrm{e}}^{[i]}} \cdot \ln \left(1+2 \cdot\left[\frac{S_{\mathrm{K}, \mathrm{r}}^{[i]}}{\left(S_{\mathrm{U}, \mathrm{r}}^{[i]}\right)^{3}}\right]^{\left[l_{\mathrm{e}}^{[i]} / l_{\mathrm{r}}\right.}-2\left[\frac{S_{\mathrm{K}, \mathrm{r}}^{[i]}}{S_{\mathrm{U}, \mathrm{r}}^{[i]}}\right]^{\left[l_{\mathrm{e}}^{[i]} / l_{\mathrm{r}}\right.}\right)= \\
& =\frac{\ln \left(S_{\mathrm{U}, \mathrm{r}}^{[i]}\right)+\ln \left(S_{\mathrm{K}, \mathrm{r}}^{[i]}\right)}{2}+\frac{l_{\mathrm{r}}}{2 \cdot l_{\mathrm{e}}^{[i]}} \cdot \ln \left(2+\left[\frac{\left(S_{\mathrm{U}, \mathrm{r}}^{[i]}\right)^{3}}{S_{\mathrm{K}, \mathrm{r}}^{[i]}}\right]^{l_{\mathrm{e}}^{[i]} / l_{\mathrm{r}}}-2 \cdot\left[\left(S_{\mathrm{U}, \mathrm{r}}^{[i]}\right)^{2}\right]^{l_{\mathrm{e}}^{[i]} / l_{\mathrm{r}}}\right) .
\end{aligned}
$$

The stress concentrations survival probability $S_{\mathrm{K}, \mathrm{r}}^{[i]}$ is calculated from Equation 18:

$$
\ln \left[S_{\mathrm{K}, \mathrm{r}}^{[i]}\left(\sigma^{\infty}\right)\right]= \begin{cases}\frac{1}{\sigma^{\infty} \cdot(k-1)} \int_{\sigma=\sigma^{\infty}}^{k \cdot \sigma^{\infty}} \ln \left[S_{\mathrm{U}, \mathrm{r}}^{[i]}(\sigma)\right] \mathrm{d} \sigma & \text { if } \quad k>1 \\ \ln \left[S_{\mathrm{U}, \mathrm{r}}^{[i]}\left(\sigma^{\infty}\right)\right] & \text { if } \quad k=1 .\end{cases}
$$

Equations 25-28 are valid for Weibull and non-Weibull strength distributions. 


\subsection{Asymptotic limits and numerical implementation}

Equations 27 and 28 show that, in order to calculate $S_{\mathrm{U}, \mathrm{r}}^{[i+1]}\left(\sigma^{\infty}\right)$, it is necessary to define $S_{\mathrm{U}, \mathrm{r}}^{[i]}\left(k \cdot \sigma^{\infty}\right)$, and thus (following a recursive procedure down to the single-fibre level) calculating $S_{\mathrm{U}, \mathrm{r}}^{[0]}\left(k^{i+1} \cdot \sigma^{\infty}\right)$. This quickly becomes intractable as bundle level increases, thus making a relevant case for an asymptotic simplification.

It can be demonstrated [23] that, if $\mathcal{C}_{\mathrm{K}}>3$ (which is verified for $m \gtrsim 2.6$ considering $k=2$, valid for most technical fibres), then Equation 27 is equivalent to the WLT (Equation 1). Each level- $[i]$ Right Tail Asympote (RTA) is defined from the single-fibre strength distribution (Equation 23) as:

$$
S_{\mathrm{u}, \mathrm{r}, \mathrm{RTA}}^{[i]}\left(\sigma^{\infty}\right)=\exp \left[-\left(\frac{\sigma^{\infty}}{\sigma_{0, \mathrm{RTA}}^{[i]}}\right)^{m_{\mathrm{RTA}}^{[i]}}\right] \text {, with }\left\{\begin{array}{l}
m_{\mathrm{RTA}}^{[i]}=m \\
\sigma_{0, \mathrm{RTA}}^{[i]}=2^{-(i+1) / m} \cdot \sigma_{0}^{\mathrm{f}}
\end{array} \quad \text { if } \quad \mathcal{C}_{\mathrm{K}} \geq 3 .\right.
$$

A comprehensive analysis of the asymptotic limits of the hierarchical scaling law can be found elsewhere [23].

An overview of the numerical implementation of the present model, which makes use of the asymptotes identified in Equation 29, is shown in Figure 5 [23]. Using array programming (e.g. MATLAB) greatly simplifies the implementation and reduces running time.

\section{RESULTS}

\subsection{Analysis of model predictions}

Figure 6 summarises the results of the model for nominal input parameters (Table 4), highlighting the size effect on bundle strength statistics in Figure 6(a). Contrarily to Newman and Gabrielov's model (Equation 3 [18]), the present one predicts an initial strengthening through bundle hierarchy (as observed experimentally $[9,11]$ ), and a steeper reduction of variability (Figure 6(a)).

The Weibull plot of bundle strength distributions in Figure 6(b) evidences a concavedown curvature for all $i>0$, although the single-fibre strength $(i=0)$ follows a Weibull distribution; this curvature initially increases for small bundles, but is progressively reduced for larger bundles within a $0.01-99.99 \%$ probability range. It is also shown that the model does converge to the WLT for large stresses, considerably before the asymptotic behaviour is mathematically imposed (Equation 29 for $\sigma^{\infty}>\sigma^{\max } / k$ ).

\subsection{Convergence study}

Figures 7 and 8 present convergence studies on the numerical variables $\sigma^{\max }$ and $\Delta \sigma$. Errors are relative to nominal inputs (Table 4), and run times were obtained with an Intel(R) Core(TM)2 Quad CPU @ $2.50 \mathrm{GHz}$, for $i^{\max }=20\left(n^{\mathrm{f}} \approx 10^{6}\right)$. It is shown that a fully converged set of strength distributions (from the single-fibre to a standard coupon-size FRP) is computed in less than a second. 
I.1: Numerical variables $\left\{\Delta \sigma, \sigma^{\max }\right\}$
I.2: Single fibre strength

$\left\{l_{\mathrm{r}}, \sigma_{0}^{\mathrm{f}}, m\right\}$
I.3: Composite bundle

$\left\{T_{\mathrm{SL}}, V^{\mathrm{f}}, \phi^{\mathrm{f}}, i^{\max }\right\}$

\section{Preliminary calculations}

II.1: Define strength vector
$n_{\boldsymbol{\sigma}}=\left\lceil\frac{\sigma^{\max }}{\Delta \sigma}\right\rceil+1$
$\boldsymbol{\sigma}=\{(j-1) \cdot \Delta \sigma\}_{j=1}^{n_{\boldsymbol{\sigma}}}$
$n_{\mathrm{K}}=\left|\frac{n_{\boldsymbol{\sigma}}-1}{k}\right|+1$

II.2: Define geometric parameters
$A^{\mathrm{f}}=\pi \cdot \frac{\left(\phi^{\mathrm{f}}\right)^{2}}{4}$
$C^{\mathrm{f}}=\pi \cdot \phi^{\mathrm{f}}$
$l_{Q}=\frac{\sqrt{\pi} \cdot \phi^{f}}{2 \cdot \sqrt{V^{f}}}$
$s_{\mathrm{Q}}=\left(\frac{\sqrt{\bar{\pi}}}{2 \cdot \sqrt{\bar{V}^{\mathrm{f}}}}-1\right) \cdot \phi^{\mathrm{f}}$

II.3: Calculate single-fibre log-survival vectors
$\operatorname{lnS}_{\mathbf{U r}}^{[0]}=-\left(\frac{\boldsymbol{\sigma}}{\sigma_{0}^{f}}\right)^{m}$
$\mathcal{C}_{\mathrm{K}}=\frac{k^{m+1}-1}{(k-1) \cdot(m+1)}$
$\ln \mathbf{S}_{\mathbf{K r}}^{[0]}=-\mathcal{C}_{\mathrm{K}}\left(\frac{\boldsymbol{\sigma}}{\sigma_{0}^{f}}\right)^{m}$

\section{Strength scaling model}

III.0: Set bundle level counter

$i=0$

$\longrightarrow i=i+1$

III.1: Define geometry and normalised effective length vector:

$n^{\mathrm{f}[i-1]}=2^{i-1} \quad C^{[i-1]}=3 \cdot C^{\mathrm{f}}+4 \cdot\left[\left(\sqrt{n^{\mathrm{f}[i-1]}}-1\right) \cdot s_{\mathrm{Q}}+\left(\sqrt{n^{\mathrm{f}[i-1]}}-2\right) \cdot C^{\mathrm{f}} / 2\right] \quad \mathbf{l}_{\mathbf{e} / \mathbf{r}}^{[i-1]}=2 \cdot \frac{n^{\mathrm{f}[i-1]} \cdot A^{\mathrm{f}}}{C^{[i-1]} \cdot T_{\mathrm{SL}} \cdot l_{\mathbf{r}}} \cdot \boldsymbol{\sigma}$

III.2: Calculate uniform-stresses bundle survival vector

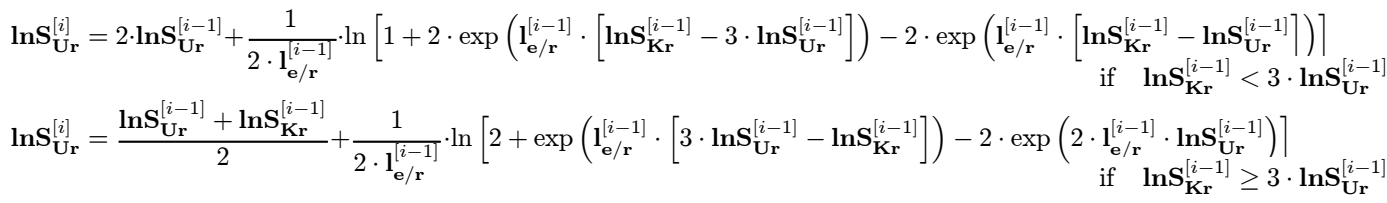

III.3: Calculate bundle survival vectors under linear stress states

$\ln \mathbf{S}_{\mathbf{L r}}^{[i]}=\frac{1}{\boldsymbol{\sigma}} \cdot \int_{\mathbf{\sigma}} \ln \mathbf{S}_{\mathbf{U r}}^{[i]} \cdot \Delta \sigma \quad \operatorname{lnS}_{\mathbf{K r}}^{[i]}(j)=\frac{k \cdot \ln \mathbf{S}_{\mathbf{L r}}^{[i]}(k \cdot j)-\ln \mathbf{S}_{\mathbf{L r}}^{[i]}(j)}{k-1} \quad$ if $\quad j \leq n_{\mathrm{K}} \quad \ln \mathbf{S}_{\mathbf{K r}}^{[i]}(j)=\mathcal{C}_{\mathbf{K}} \cdot \ln \mathbf{S}_{\mathbf{U r}}^{[i]}(j) \quad$ if $\quad j>n_{\mathrm{K}}$

\section{Post-processing}

IV.0: Set bundle level counter

$i=0$

$\longrightarrow i=i+1$

IV.1: Calculate bundle strength distributions

$\mathbf{F}_{\mathbf{U r}}^{[i]}=1-\exp \left[\operatorname{lnS}_{\mathbf{U r}}^{[i]}\right]$

IV.2: Calculate bundle strength statistics

$X_{\mathrm{m}}^{[i]}=\boldsymbol{\sigma}\left(n_{\boldsymbol{\sigma}}\right)-\int_{\mathbf{\sigma}} \mathbf{F}_{\mathbf{U} \mathbf{r}}^{[i]} \cdot \Delta \sigma$

$$
\operatorname{CoV}_{X}^{[i]}=\frac{\sqrt{\boldsymbol{\sigma}\left(n_{\mathbf{\sigma}}\right)^{2}-\left(X_{\mathrm{m}}^{[i]}\right)^{2}-2 \cdot \int_{\mathbf{\sigma}} \boldsymbol{\sigma} \cdot \mathbf{F}_{\mathrm{Ur}}^{[i]} \cdot \Delta \sigma}}{X_{\mathrm{m}}^{[i]}}
$$

if $i<i^{\max }$

Figure 5. Numerical implementation.

Table 4. Nominal inputs for parametric studies (nominal outputs to be highlighted as $\diamond$ ).

\begin{tabular}{|c|c|c|c|c|c|c|c|c|}
\hline \multicolumn{2}{|c|}{ Numerical parameters } & \multicolumn{3}{|c|}{ Mechanical properties } & \multicolumn{3}{|c|}{ Geometry } & \multirow{2}{*}{$\begin{array}{c}\text { Load } \\
k\end{array}$} \\
\hline$\sigma^{\max }(\mathrm{GPa})$ & $\Delta \sigma(\mathrm{MPa})$ & $X_{\mathrm{m}}^{\mathrm{f}}(\mathrm{GPa})$ & $\operatorname{CoV}_{X}^{\mathrm{f}}(\%)$ & $T_{\mathrm{SL}}(\mathrm{MPa})$ & $\phi^{\mathrm{f}}(\mu \mathrm{m})$ & $V^{\mathrm{f}}(\%)$ & $\Gamma_{\mathrm{SL}}$ & \\
\hline 50 & 1 & 4.5 & 25 & 70 & 5 & 60 & QI & 2 \\
\hline
\end{tabular}




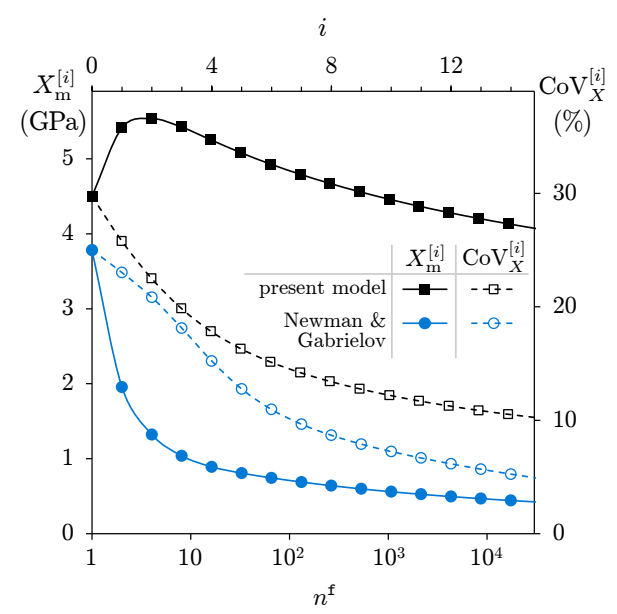

(a) Bundle strength size effect: present model vs. Newman and Gabrielov's [18].

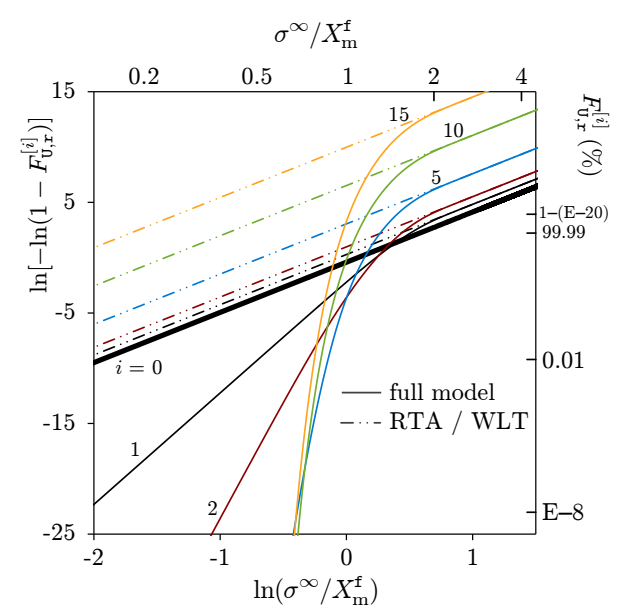

(b) Weibull plots for predicted bundle strength distributions.

Figure 6. Overview of model results.

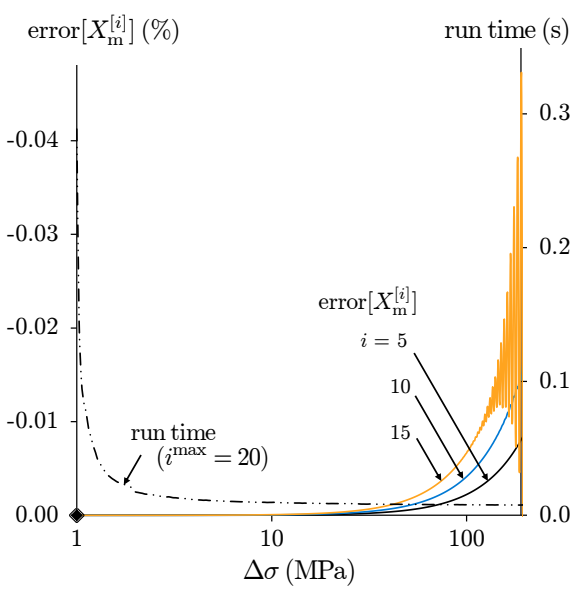

(a) Mean bundle strength.

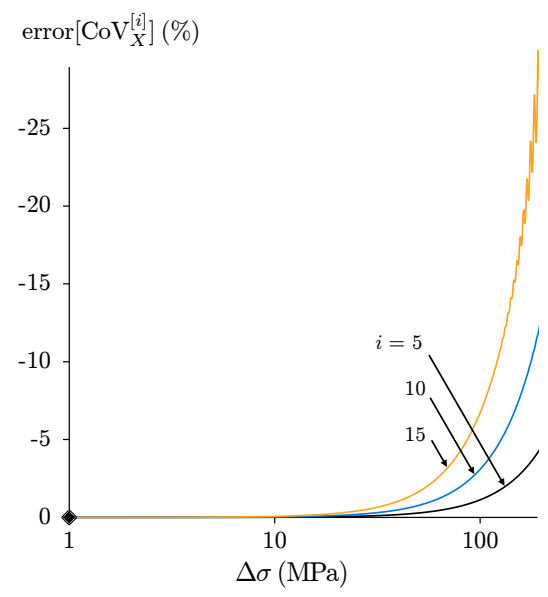

(b) Bundle strength $\mathrm{CoV}$.

Figure 7. Convergence of bundle strength statistics with integration step (for several levels $i$ ).

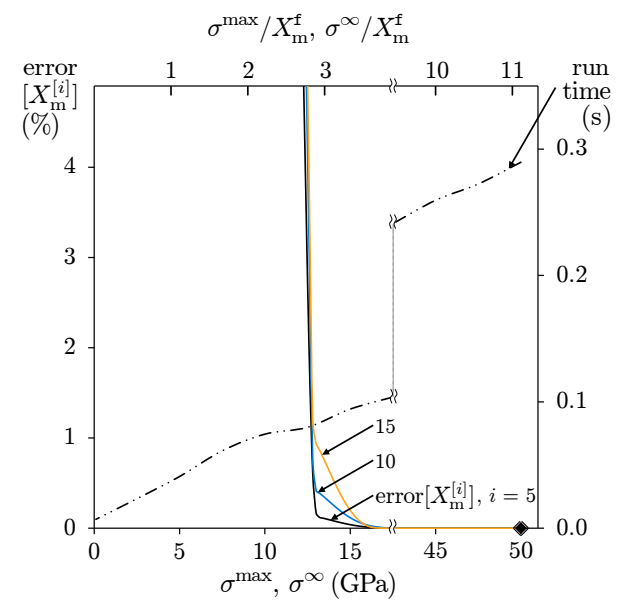

(a) Mean bundle strength.

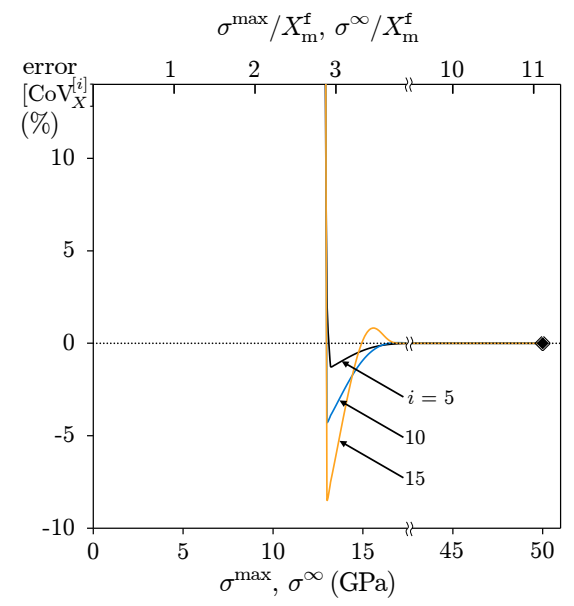

(b) Bundle strength CoV.

Figure 8. Convergence of bundle strength statistics with upper integration limit (for several levels $i$ ). 


\subsection{Parametric study}

The relation between single-fibre strength variability and bundle strength distributions is shown in Figure 9. For a deterministic fibre strength, the model predicts no size effects; for a small $\mathrm{CoV}_{X}^{\mathrm{f}}$, the model converges to the WLT (Equation 29).

Figure 10 shows that increasing the shear-lag strength strengthens the bundles and reduces their variability. For very low $T_{\mathrm{SL}}$ values, size effects converge to the WLT (Equation 29) and, therefore, mean bundle strength decreases monotonically with bundle level.

Matching several $\Gamma_{\mathrm{SL}}$ geometries (Section 2.2, Figure 11(a)) with the corresponding mean bundle strength (Figure 11(b)) reveals that the largest shear-lag boundary at low-level bundles (QM geometry) yields the strongest bundles throughout the whole hierarchy; the same is not verified for the largest shear-lag boundary at high-level bundles (HI geometry). Freeboundary effects (QB vs. QI geometries) affect small bundles only.

\subsection{Validation against experimental results}

Beyerlein and Phoenix [9] and Kazanci [11] measured the strength distributions of several micro-composites (detailed description in Tables 5 and 6). Figure 12 compares experimental results to model predictions; each plot shows the strength distribution of the singlefibre and the two corresponding bundles (manufactured with different resin systems), as well as predictions from the present and Newman and Gabrielov's [18] models.

Okabe and Takeda [12] analysed size effects on the strength of a Toray T800H/3631 (carbon-epoxy) system (Tables 5 and 6 ) by testing $10 \mathrm{~mm}$ long bundles with $10^{4}-10^{6}$ fibres. Figure 13 shows the experimentally measured bundle strengths, together with the strength probability map predicted by the model for two values shear-lag strengths; Newman and Gabrielov's [18] mean bundle strengths are shown for comparison.

Table 5. Description of composites for model validation.

\begin{tabular}{ccccccc}
\hline Composite ref. & Fibre ref. ${ }^{(\star)}$ & $n^{\text {f }}$ & Matrix ref. $\left.{ }^{(\dagger}\right)$ & $T_{\mathrm{SL}}(\mathrm{MPa})$ & $V^{\mathrm{f}}$ & Reference \\
\hline $\mathrm{A}^{4} \mathrm{~S}$ & $\mathrm{~A}$ & 4 & $\mathrm{~S}$ & $46.6^{(\S)}$ & $70 \%$ & {$[9]$} \\
$\mathrm{A}^{4} \mathrm{~F}$ & $\mathrm{~A}$ & 4 & $\mathrm{~F}$ & $10.3^{(\S)}$ & $70 \%$ & {$[9]$} \\
$\mathrm{I}^{7} \mathrm{~S}$ & $\mathrm{I}$ & 7 & $\mathrm{~S}$ & $46.6^{(\S)}$ & $56 \%$ & {$[11]$} \\
$\mathrm{I}^{7} \mathrm{~F}$ & $\mathrm{I}$ & 7 & $\mathrm{~F}$ & $10.3^{(\S)}$ & $56 \%$ & {$[11]$} \\
\hline $\mathrm{T}^{\mathrm{n}} \mathrm{T}$ & $\mathrm{T}$ & $10^{4}-10^{6}$ & $\mathrm{~T}$ & 52.4 & $60 \%$ & {$[12]$} \\
\hline
\end{tabular}

(*) See Table 6 for detailed description.

(†) Epoxy resins. Standard (S): DER 331, Dow Plastics; Flexible (F): DER 331 + DER 732 (50:50), Dow Plastics; Toughened (T): 3631, Toray Composites.

(§) Drucker-Prager's criterion, using tensile and compressive strengths [24].

Table 6. Carbon-fibre data for model validation.

\begin{tabular}{ccccccc}
\hline Fibre ref. & Fibre type $^{(\star)}$ & $\phi^{\mathrm{f}}(\mu \mathrm{m})$ & $l_{\mathrm{r}}^{\mathrm{f}}(\mathrm{mm})$ & $m^{(\dagger)}$ & $\sigma_{0}^{\mathrm{f}}\left({ }^{\dagger}\right)(\mathrm{GPa})$ & Reference \\
\hline A & AS4 & 6.85 & 10 & 4.8 & 4.493 & {$[9]$} \\
I & IM6 & 5.63 & 10 & 5.4 & 5.283 & {$[11]$} \\
\hline T & T800 & 5.00 & 50 & 3.8 & 3.570 & {$[12]$} \\
\hline
\end{tabular}

${ }^{\star} \star$ A AS4 and IM6 fibres provided by Hercules / Hexcel; T800 fibres provided by Toray.

(†) From the respective Reference, originally calculated through the maximum likelihood method. 


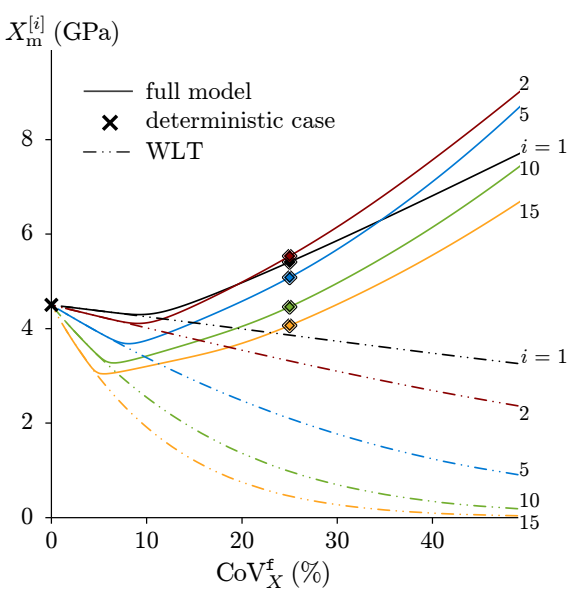

(a) Mean bundle strength.

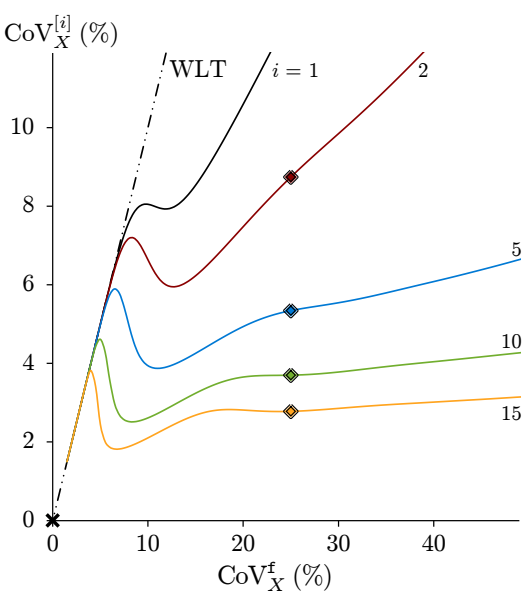

(b) Bundle strength $\mathrm{CoV}$.

Figure 9. Effect of the $\mathrm{CoV}$ of single-fibre strength on bundle strength statistics (for several levels $i$ ).

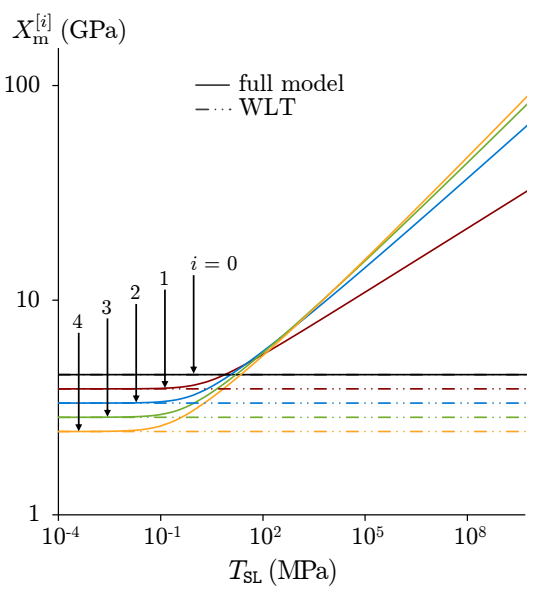

(a) Mean bundle strength.

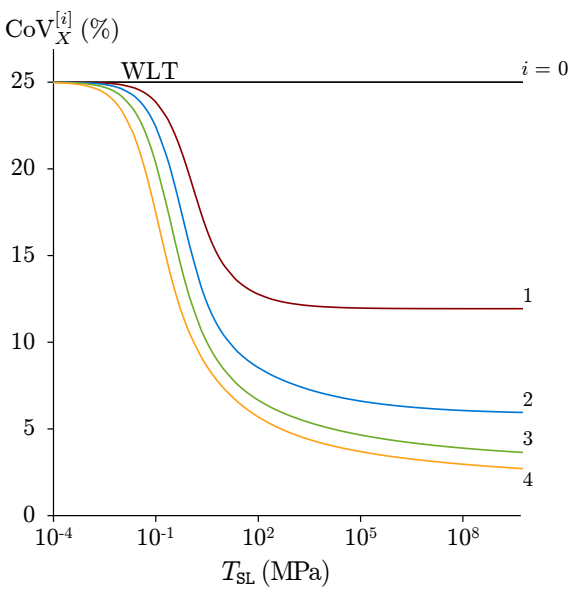

(b) Bundle strength CoV.

Figure 10. Effect of shear-lag strength $\left(T_{\mathrm{SL}}\right)$ on bundle strength statistics (for several levels $i$ ).

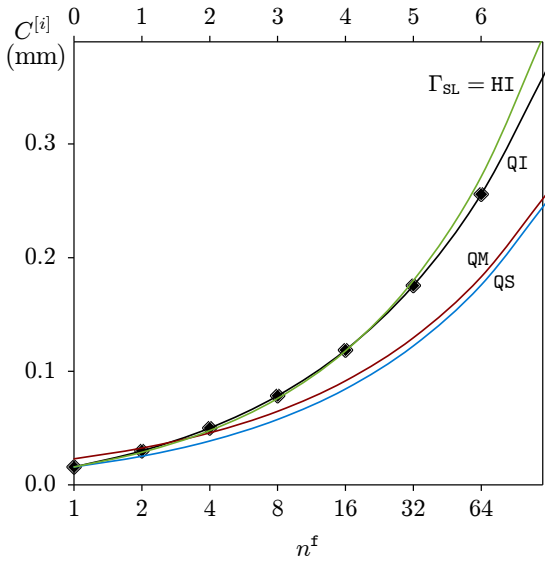

(a) Shear-lag boundary perimeter.

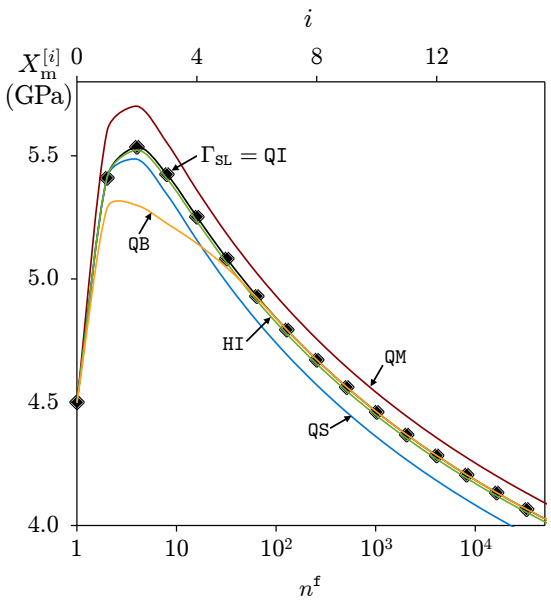

(b) Mean bundle strength.

Figure 11. Bundle strength size effect for several shear-lag geometries $\left(\Gamma_{\mathrm{SL}}\right)$. 


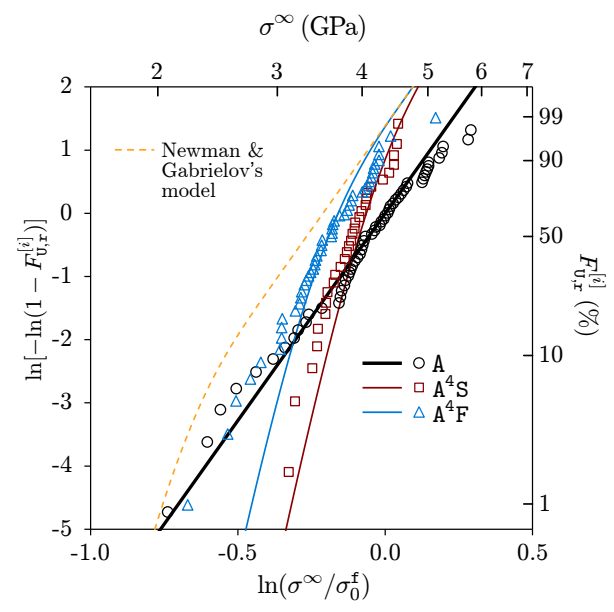

(a) AS4 fibre type

$\left(m=6.6, \sigma_{0}^{\mathrm{f}}=4.3 \mathrm{GPa}\right.$ at $\left.l_{\mathrm{r}}=10 \mathrm{~mm}\right)$.

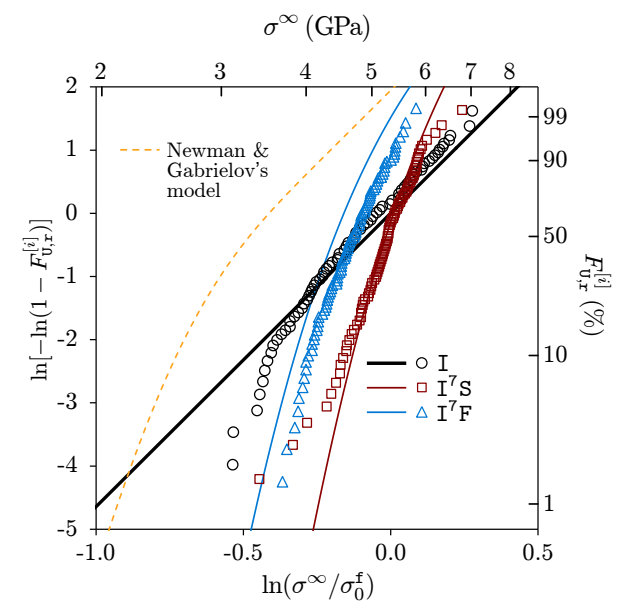

(b) IM6 fibre type

$\left(m=4.6, \sigma_{0}^{\mathrm{f}}=5.3 \mathrm{GPa}\right.$ at $\left.l_{\mathrm{r}}=10 \mathrm{~mm}\right)$.

Figure 12. Micro-composite strength distributions: experimental results (data points $[9,11]$ ), visually fitted single-fibre distribution for model input (thick lines), present model predictions for both resins types (thin lines), and Newman and Gabrielov's [18] prediction (dashed lines).

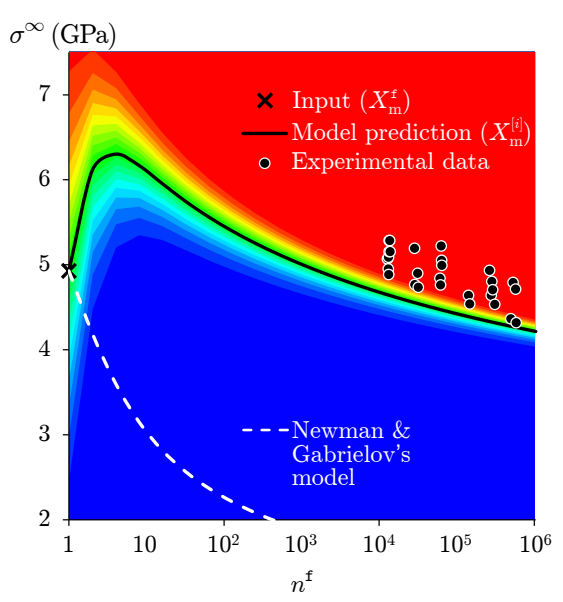

(a) $T_{\mathrm{SL}}=52.4 \mathrm{MPa}$ (nominal).

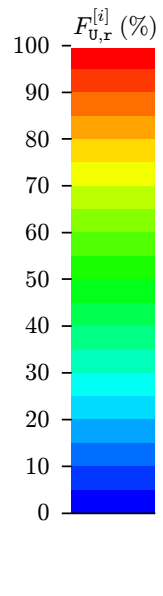

Figure 13. Macro-bundle strengths for model validation: experimental bundle strengths (data points [12]), present model's probability map (with mean strength highlighted), and Newman and Gabrielov's [18] mean strength prediction (dashed line in (a)).

\section{DISCUSSION}

\subsection{Features captured by the model}

The model captures many characteristic features of strength size-effects in FRPs:

a. Size effects result from fibre strength variability (Figure 9), which leads to the WLT for damage initiation. Damage propagation is arrested by the presence of the matrix / interface, which limits stress concentrations and coalescence of fibre-breaks.

b. Both the magnitude of the size effect and the variability of tensile strength decrease with increasing specimen size (Figure 6(a)) [1]. 
c. The presence of matrix / interface is most relevant for micro-bundles ( $i \lesssim 3$, Figure 11), resulting in the latter being stronger than their constituent fibres (for reasonably strong matrices). The effect of fibre strength variability is dominant in large bundles ( $n^{\mathrm{f}} \gtrsim 50$ ).

d. Predicted large-bundle strength distribution appear quasi-linear in a Weibull plot within a 0.01-99.99\% probability range (Figure 6(b), $i=15$ ), which explains the good agreement usually reported between the Weibull-based WLT and experiments [1].

\subsection{Relation between proposed model and WLT}

The present model's formulation differs from the WLT by considering stable modes of bundle failure; these are mathematically represented in the strength scaling law (Equation 25) by the second term on the right hand side. Consequently, the model degenerates into the WLT whenever these failure modes cannot take place (i.e. for low $\mathrm{CoV}_{X}^{\mathrm{f}}$ (Figure 9), $T_{\mathrm{SL}} \rightarrow 0$ (Figure 10), and $k \rightarrow \infty$ ). A comprehensive discussion on the asymptotic limits of bundle strength distribution for extreme values of these variables is presented elsewhere [23].

Figure 14 compares bundle strength statistics obtained by either (i) running the full model for all bundle levels $i$, or (ii) running the model up to level $i^{\mathrm{WLT}}$ followed by the WLT (Equation 1) for each level $i>i^{\mathrm{WLT}}$. Although the WLT applied directly from the single-fibre $\left(i^{\mathrm{WLT}}=0\right)$ severely underestimates mean strengths and overestimates CoVs, both approaches converge if applied from a certain bundle level onwards ( $i^{\mathrm{WLT}} \gtrsim 5$ in this case). This indicates that the model captures the quasi-brittle nature of FRPs $[1,12,13]$.

\subsection{Effect of the shear-lag boundary and relation to Newman and Gabrielov's model}

By comparing the present model and Newman and Gabrielov's one [18] (Figure 6(a)), it is highlighted that considering the matrix / interface significantly increases mean bundle strength and reduces variability. As shown in Figure 11, this affects directly small bundles $(i \lesssim 3)$ only, but the effect propagates throughout bundle hierarchy.

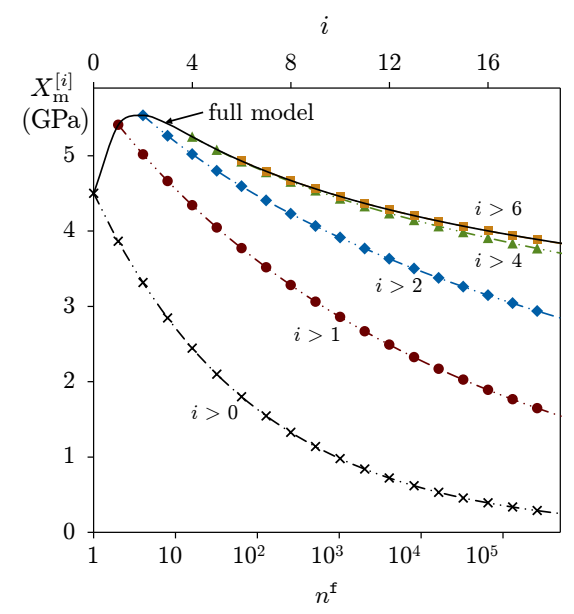

(a) Mean bundle strength.

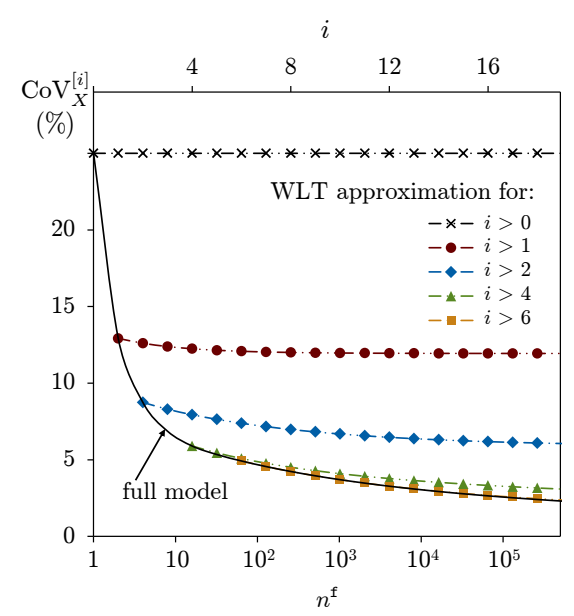

(b) Bundle strength $\mathrm{CoV}$.

Figure 14. Bundle strength size effect considering a WLT approximation for large bundles. 
Including the effect of the shear-lag boundary results in considering the length of the damage process zone $\left(l_{c}^{[i]}\right)$, which is fundamental for defining size effects in quasi-brittle materials [3]. Such feature not only improved the accuracy of model predictions (Figures 12 and 13), as it makes bundle strength scaling independent of the reference length $l_{r}$ used in the calculations [23].

\subsection{Model inputs and experimental validation}

Despite the difficulties associated with determining correct input properties (especially regarding single-fibre strength distributions [10] and in-situ matrix properties [19]), the comparison between model predictions and experimental data available in the literature (Figures 12 and 13) is extremely encouraging.

In Figure 12, the model evidences its ability to reproduce quantitatively the effect of different fibres and resins on micro-bundle strength distributions; this offers a strong support to the shear-lag approach used. The model reproduces the concave-down curvature of bundle strength distributions, the different slopes and locations of the four data sets, and the relative orientations within each pair of data for the same fibre type (converging right tails in Figure 12(a), nearly parallel distributions in 12(b)).

Figure 13 considers bundles with cross sections up to $30 \mathrm{~mm}^{2}$ (larger than the standard UD FRP specimen for characterisation of tensile properties). A very good agreement between predicted and measured strengths is obtained when increasing the input value of shear-lag strength (Figure 13(b)), which is likely to be more representative of the true in-situ matrix behaviour. The reduction of strength variability for larger bundles is captured as well, and nearly all the experimental scatter is covered within the predicted 5-95\% percentiles for bundle strength distributions.

\section{CONCLUSIONS}

An analytical model for size effects on the longitudinal tensile strength of FRP bundles was developed, implemented and validated. The model is based on the stochastic analysis of the failure process in hierarchical fibre bundles, considering Weibull fibres and a simplified shear-lag model to represent matrix effects.

The model predicts full strength distributions and statistics for bundles of any size. The matrix (or fibre-matrix interface) was shown to have a significant strengthening effect, which supports the present model over others not including this feature [2,18]. An efficient numerical scheme was proposed, leading to full-model running times below one second.

The model was validated both at the micro and macro scales, showing a remarkable agreement with experimentally measured bundle strengths in a large range of sizes. The quasibrittle nature of composites is reproduced; the model also illustrates many experimentally observed trends, such as the tensile strength of FRPs appearing to follow a Weibull distribution, and large-scale size effects consistent with the WLT.

Predictive models for size effects in composite materials are paramount for scaling small-coupon experimental results to the design of large structures. In addition to such quantitative predictions, the present work provides insight on the longitudinal tensile failure pro- 
cess. The model's ability to compute strength distributions for small bundles (rather than only for asymptotically large ones) makes it particularly suitable for state-of-the-art multiscale discontinuous-fibre composites. Further developments will include predicting the shape of fracture surfaces and the corresponding fracture toughness for FRPs under longitudinal tensile failure.

\section{Acknowledgements}

The funding from the Portuguese Foundation for Science and Technology (project nr. SFRH/BD/44051/2008) is gratefully acknowledged.

\section{REFERENCES}

[1] M. R. Wisnom, "Size effects in the testing of fibre-composite materials," Composites Science and Technology, 59, 1937-1957, 1999.

[2] G. W. Weibull, "A statistical distribution function of wide applicability," Journal of Applied Mathematics, 293-297, 1951.

[3] Z. P. Bažant, "Size effect on structural strength: a review," Archive of Applied Mechanics, 69, 703-725, 1999.

[4] A. Carpinteri, "Scaling laws and renormalization-groups for strength and toughness of disordered materials," International Journal of Solids and Structures, 31, 291-302, 1994.

[5] M. R. Wisnom, S. R. Hallett, and C. Soutis, "Scaling effects in notched composites," Journal of Composite Materials, 44, 195-210, 2010.

[6] L. Mishnaevsky and P. Brondsted, "Micromechanical modeling of damage and fracture of unidirectional fiber reinforced composites: A review," Computational Materials Science, 44, 1351-1359, 2009.

[7] S. Pradhan, A. Hansen, and B. K. Chakrabarti, "Failure processes in elastic fiber bundles," Reviews of Modern Physics, 82, 499-555, 2010.

[8] C. P. Beetz, "The analysis of carbon-fiber strength distributions exhibiting multiplemodes of failure," Fibre Science and Technology, 16, 45-59, 1982.

[9] I. J. Beyerlein and S. L. Phoenix, "Statistics for the strength and size effects of microcomposites with four carbon fibers in epoxy resin," Composites Science and Technology, 56, 75-92, 1996.

[10] E. G. Stoner, D. D. Edie, and S. D. Durham, "An end-effect model for the singlefilament tensile test," Journal of Materials Science, 29, 6561-6574, 1994.

[11] M. Kazanci, "Carbon fiber reinforced microcomposites in two different epoxies," Polymer Testing, 23, 747-753, 2004. 
[12] T. Okabe and N. Takeda, "Size effect on tensile strength of unidirectional CFRP composites - experiment and simulation," Composites Science and Technology, 62, 20532064, 2002.

[13] A. E. Scott, M. Mavrogordato, P. Wright, I. Sinclair, and S. M. Spearing, "In situ fibre fracture measurement in carbon-epoxy laminates using high resolution computed tomography," Composites Science and Technology, 71, 1471-1477, 2011.

[14] L. T. Harper, T. A. Turner, N. A. Warrior, and C. D. Rudd, "Characterisation of random carbon fibre composites from a directed fibre preforming process: The effect of tow filamentisation," Composites Part A - Applied Science and Manufacturing, 38, 755770, 2007.

[15] S. Pimenta, S. T. Pinho, P. Robinson, K. H. Wong, and S. J. Pickering, "Mechanical analysis and toughening mechanisms of a multiphase recycled CFRP," Composites Science and Technology, 70, 1713-1725, 2010.

[16] H. E. Daniels, "The statistical theory of the strength of bundles of threads. I," Proceeedings of the Royal Society A - Mathematical, Physical and \& Engineering Science, 183, 405-435, 1945.

[17] M. J. Laffan, S. T. Pinho, P. Robinson, and L. Iannucci, "Measurement of the in situ ply fracture toughness associated with mode I fibre tensile failure in FRP. Part II: Size and lay-up effects," Composites Science and Technology, 70, 614-621, 2010.

[18] W. I. Newman and A. M. Gabrielov, "Failure of hierarchical distributions of fiberbundles. 1," International Journal of Fracture, 50, 1-14, 1991.

[19] T. Hobbiebrunken, B. Fiedler, M. Hojo, and M. Tanaka, "Experimental determination of the true epoxy resin strength using micro-scaled specimens," Composites Part AApplied Science and Manufacturing, 38, 814-818, 2007.

[20] A. B. de Morais, "Stress distribution along broken fibres in polymer-matrix composites," Composites Science and Technology, 61, 1571-1580, 2001.

[21] M. R. Nedele and M. R. Wisnom, "3-dimensional finite-element analysis of the stressconcentration at a single-fiber break," Composites Science and Technology, 51, 517524, 1994.

[22] I. J. Beyerlein and S. L. Phoenix, "Stress concentrations around multiple fiber breaks in an elastic matrix with local yielding or debonding using quadratic influence superposition," Journal of the Mechanics and Physics of Solids, 44, 1997-2039, 1996.

[23] S. Pimenta and S. T. Pinho, "Hierarchical scaling law for the strength of composite fibre bundles," submitted to the Journal of the Mechanics and Physics of Solids, 2012.

[24] Dow Plastics, "Dow liquid epoxy resins," Form No. 296-00224-0199 WC+M., http: //epoxy.dow.com/epoxy/tech/index.htm, last accessed on 29 January $2011,1999$. 